\title{
Physical interaction between tip and molecules in scanning force microscopy imaging of adsorbed $\mathrm{C}_{60}$ and fullerene tubules
}

\author{
Alain Dereux \\ Institute for Studies in Interface Sciences, Facultés Universitaires Notre-Dame de la Paix, \\ Rue de Brixelles 6J, B-5000 Namur, Belgium \\ Christian Girard \\ Laboratoire de Physique Moléculaire, Université de Franche-Comté, F-25030 Besançon cedex, France \\ Olivier J. F. Martin \\ Swiss Federal Institute of Technology ETH, Institute for Field Theory, Gloriastr. 35, \\ $\mathrm{CH}-8092$ Zürich. Switzerland \\ Philippe Lambin and Henning Richter \\ Institute for Studies in Interface Sciences, Facultés Universitaires Notre-Dame de la Paix, \\ Rue de Bruxelles 61, B-5000 Namur, Belgium
}

(Received 26 July 1994; accepted 7 September 1994)

After the discovery of $\mathrm{C}_{60}$, a large family of fullerene molecules was also identified. Among them, elongated fullerenes are formed by the tubular assembly of carbon atoms. The van der Waals bonds between fullerene molecules are due to the correlations between fluctuating charge densities inside the molecules. The interaction is then dominated by collective excitations which are sensitive to the shape of the molecules. Therefore, van der Waals attraction is expected to be modified when considering successively spherical $\mathrm{C}_{60}, \mathrm{C}_{70}$ and more elongated fullerenes (tubules). This paper presents self-consistent computations of the van der Waals interaction between a (111) diamond probe tip and various fullerene molecules adsorbed on a gold surface. Relative to spherical $\mathrm{C}_{60}$, the dependence law of the force experienced by the probe tip as a function of the tip-sample distance decreased when approaching fullerene tubules. Simulations of scanning force microscope scans of carbon tubules next to $\mathrm{C}_{60}$ molecules show that the shape of the molecules affects the interpretation of scanning force microscopy imaging. Particularly, information about the height of the various molecules deposited on the surface must be considered with some care since carbon tubules with the same radius as $\mathrm{C}_{60}$ interact more strongly with the probe tip. (c) 1994 American Institute of Physics.

\section{INTRODUCTION}

In addition to its ability to provide atomically resolved images of surfaces, scanning force microscopy (SFM) ${ }^{1}$ enables one to observe molecules adsorbed on these surfaces. In the context of the rapidly developing research on fullerene molecules, SFM studies of a submonolayer coverage of $\mathrm{C}_{60}$ deposited on metallic surfaces were reported. ${ }^{2,3}$ SFM was also used to investigate surfaces of heterogeneous crystal made of $\mathrm{C}_{60}$ and $\mathrm{C}_{70} \cdot{ }^{4}$ Nanoscale friction measurements of $\mathrm{C}_{60}$ adsorbed on silicon and mica were performed and imaged using a SFM. The perspective of controlled manipulations of individual fullerene molecules demands a deep understanding of the interaction which is responsible for the imaging process of fullerene molecules by SFM tips. ${ }^{5}$ The successful experimental steps taken recently to study the transfer of charges through the intramolecular tunneling channels of $\mathrm{C}_{60}$ deposited on gold made this last perspective more vivid. ${ }^{6}$

The interpretation of SFM images depends critically on the physical interaction between tip and sample. ${ }^{7}$ Scanning at very short distances involves mainly a repulsive force due to the overlap of electron clouds. This force may be anisotropic and is very sensitive to tiny changes of the tip-sample distance. At larger distances, only the van der Waals force makes possible the interaction between electrically neutral objects. This long-range force is due to the correlations be- tween the fluctuating charge densities inside each object. In this experimental setup, the interaction is then dominated by the collective excitations of the charge density. When observing fullerene molecules by SFM, experiments seemed to indicate that the importance of these collective excitations prevents one from reaching intramolecular resolution although the practical device was able to resolve naked crystal surfaces atomically. SFM being sensitive to the tip-sample forces, the recorded images revealed the structure which was responsible for these forces, namely the spatial distribution of the total charge spread inside each fullerene molecules which was interacting collectively with the charge density of the probe tip.

Supported by self-consistent computations of the van der Waals interaction between a diamond tip and different fullerene molecules, the present work points out that the collective excitations which build the dispersion energy are sensitive to the shape of the molecules. After a summary of the analytical framework, computations of SFM scans above a cluster of adsorbed $\mathrm{C}_{60}$ are presented to assess how the van der Waals interaction is affected by the introduction of a lacuna or a $\mathrm{C}_{70}$ molecule. Numerical simulations will next show that the van der Waals attraction is modified when approaching various fullerene tubules having the same radius. 


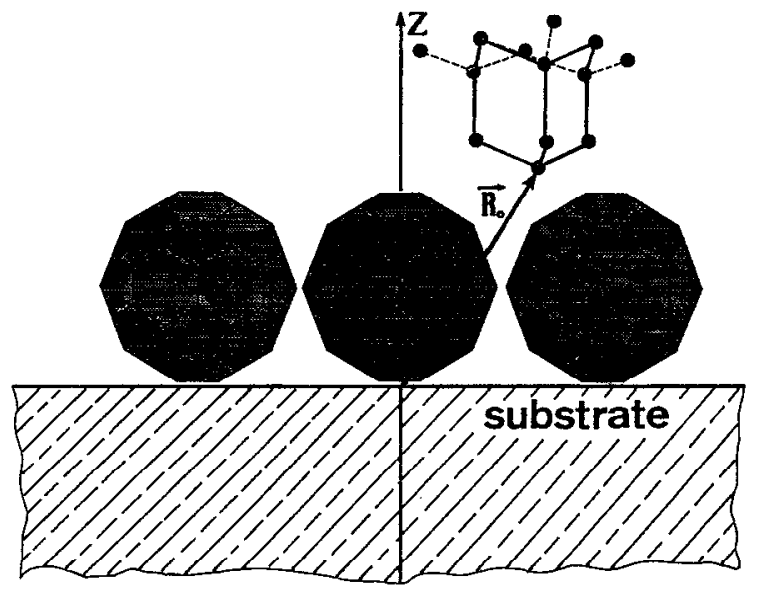

FIG. 1. Sehematic representation of a (111) diamond tip interacting with several fullerene molecules. The vector $\mathbf{R}_{0}=\left(X_{0}, Y_{0}, Z_{0}\right)$ defines the position of the atom located at the tip apex.

\section{INTERACTION BETWEEN SFM TIP AND ADSORBED MOLECULES}

Simulating SFM images requires a specific model of the tip. In this work, the discrete structure of the tip apex is composed of at least four layers of carbon atoms. Their arrangement follows the $C_{3 \nu}$ symmetry of diamond tips (Fig. 1). The adequacy of using such a tip model for SFM scans simulations to reproduce experimental images of graphite was assessed recently. ${ }^{8}$ At very short tip-sample distances, the interaction is repulsive. The model described below accounts for this contribution through pairwise interactions of the form $\left(\mathbf{r}_{i}\right.$ locates an atomic position)

$$
U_{r}\left(\mathbf{r}_{i}, \mathbf{r}_{j}\right)=\frac{K_{12}}{\left|\mathbf{r}_{i}-\mathbf{r}_{j}\right|^{12}}
$$

At larger distances, the long-range attractive van der Waals interaction dominates. This interaction arises from the electric correlations between fluctuating dipole moments of atoms inside the tip and the molecules. The collective excitations of the system provides an efficient framework to de-
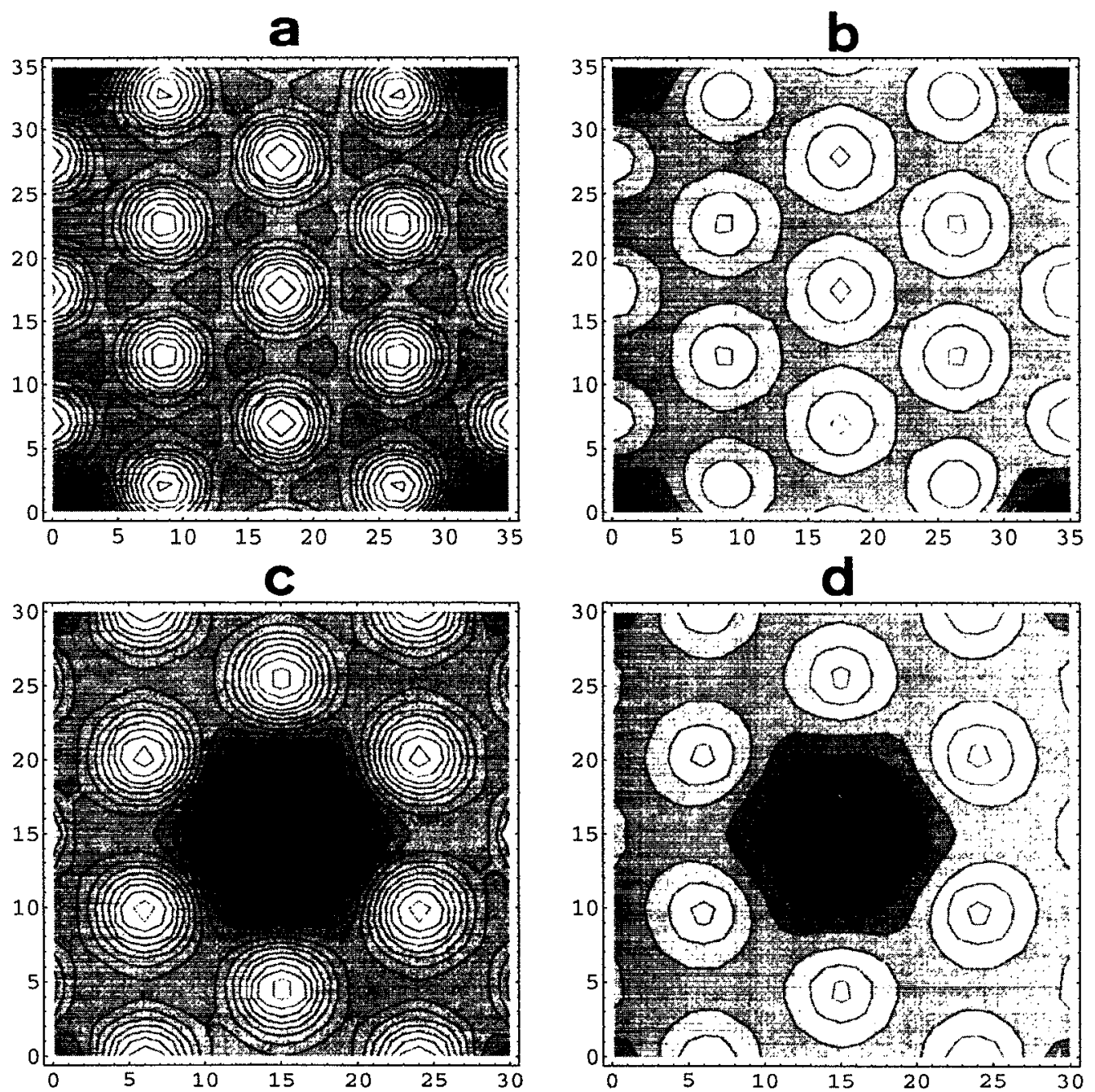

FIG. 2. Constant-distance images of hexagonally arranged $\mathrm{C}_{60}$ islands adsorbed on a plane gold surface. The contour levels vary by step of $10 \mathrm{pN}$ while the gray scale grows from black to white. The calculations were performed in planes parallel to the surface defined by: (a) $Z_{0}=14 \AA$ : the force varies from 50 to $190 \mathrm{pN}$, (b) $Z_{0}=15 \AA$ : the force varies from 30 to $90 \mathrm{pN}$, (c) same as (a) but a lacuna was created by removing the central molecule, and (d) same as (b) with a lacuna at the center of the cluster. Above the lacuna, the lowest levels (black) correspond respectively to $50 \mathrm{pN}$ (c) and $30 \mathrm{pN}$ (d). 

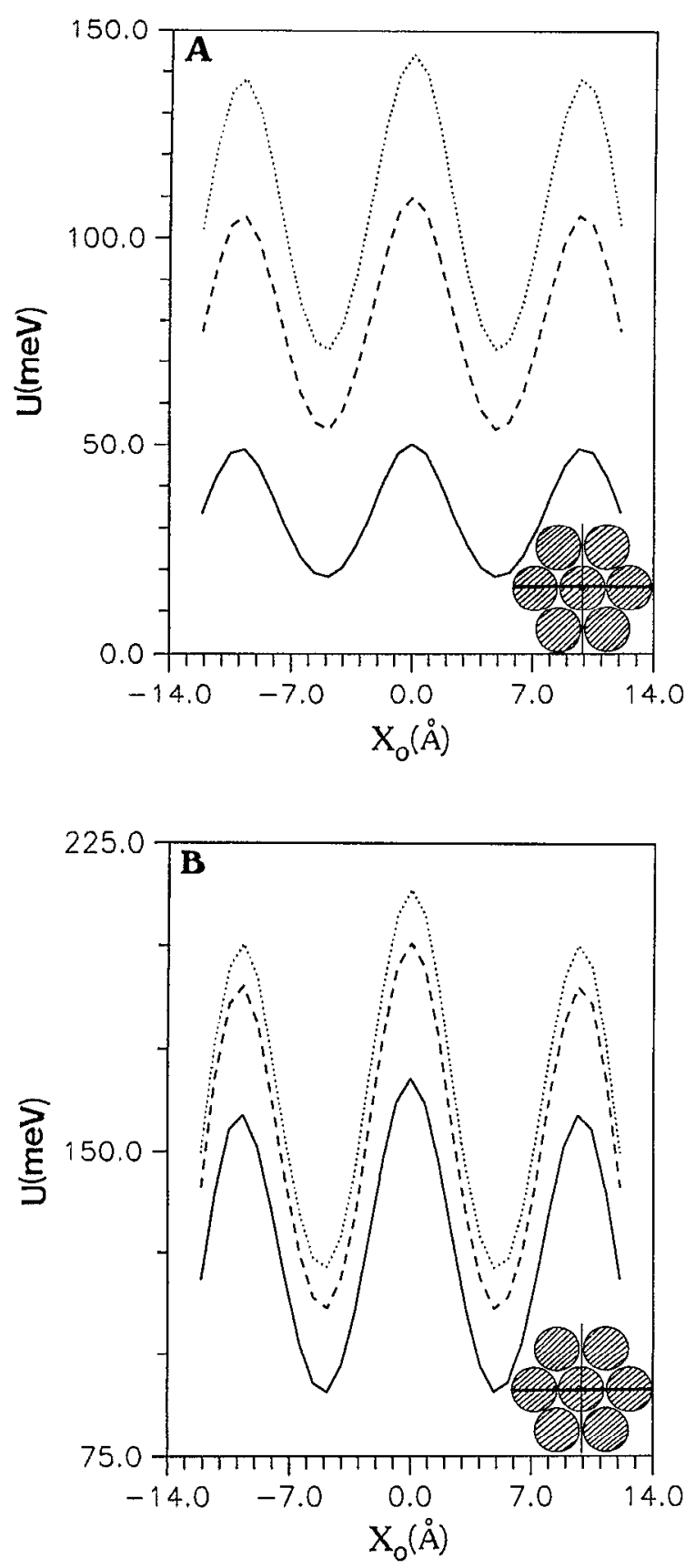

FIG. 3. For the approach distance $Z_{0}=14 \AA$, evolution of the tip-sample interaction energy during a scan above an island of seven $\mathrm{C}_{60}$ molecules as a function of the size of the diamond tip. The atoms of the second layers are located in plane located $0.514 \AA$ above the single atom at the apex. This same distance separates any even layer from the underlying odd layer while the atoms belonging to any odd layer are located $2.04 \AA$ above the preceding even layer. (a) The tip is successively made of a single $C$ atom (solid line), $4 \mathrm{C}$ atoms (dashed line), and $7 \mathrm{C}$ atoms (dotted line). (b) The number of $\mathrm{C}$ layers in the diamond tip grows from 4 to $6: 13$ atoms (solid line), 19 atoms (dashed line), and 29 atoms (dotted line).

scribe the dispersion energy between a SFM probe tip and a sample surface. Indeed, the formulation is then focused on the coupled electromagnetic modes which are primarily responsible for the dispersion energy. ${ }^{9}$ Moreover, it provides a tractable approach to deal with the underlying many-body effects. The remainder of this section summarizes the ana- lytical framework which supports our numerical computations of this attractive van der Waals interaction.

The fullerene molecules and the tip are viewed as rigid clusters of polarizable $\mathrm{C}$ atoms. The molecules are adsorbed on a model surface which is simply a semi-infinite continuous dielectric medium with a spatially local response. A fluctuating electric field $\mathbf{E}(\mathbf{r}) e^{-i \omega t}$ induces dipole moments on each atom $\left[\alpha_{i}(\omega)\right.$ is the polarizability of the $i$ th $\mathrm{C}$ atom $\left.{ }^{10}\right]$ :

$$
\mathbf{p}_{i}=\alpha_{i}(\omega) \quad \mathbf{E}\left(\mathbf{r}_{i}\right) .
$$

The resulting dipoles generate an electric field according to ( $N$ is the number of $\mathrm{C}$ atoms, including those of the tip)

$$
\mathbf{E}\left(\mathbf{r}_{i}\right)=\sum_{j=1}^{N} S_{0}\left(\mathbf{r}_{i}, \mathbf{r}_{j}, \omega\right) \mathbf{p}_{j},
$$

where $S_{0}\left(\mathbf{r}_{i}, \mathbf{r}_{j} ; \omega\right)$ is the so-called field propagator which gives the electric field at $\mathbf{r}_{i}$ due to a dipole located at $\mathbf{r}_{j}$ above the naked substrate. Details on the analytical construction of $S_{0}\left(\mathbf{r}_{i}, \mathbf{r}_{j} ; \omega\right)$ may be found in earlier works. "The relation (2) allows us to establish the self-consistency of the fluctuating field $\mathbf{E}\left(\mathbf{r}_{i}\right)$ in the form of a linear set of equations:

$\mathbf{E}\left(\mathbf{r}_{i}\right)-\sum_{j=1}^{N} S_{0}\left(\mathbf{r}_{i}, \mathbf{r}_{j} ; \omega\right) \quad \alpha_{j}(\omega) \quad \mathbf{E}\left(\mathbf{r}_{j}\right)=0, \quad i=1, \ldots N$.

For a given configuration of the tip (labeled by $\mathbf{R}_{0}$, see Fig. 1), the coupled tip-sample system sustains eigenmodes at frequencies $\omega$ given by the zeros of the determinant $\Delta\left(\omega, \mathbf{R}_{0}\right)$ of the $3 N \times 3 N$ scattering matrix defined by Eq. (4). Quantized eigenmodes possess zero-point energies which are added to each other to give the van der Waals energy. A classical theorem of complex analysis allows to transform this sum over positive zeros into the following integral over the imaginary axis which is better designed for numerical purposes: ${ }^{12}$

$$
U_{d}\left(\mathbf{R}_{0}\right)--\frac{\hbar}{2 \pi} \int_{0}^{+\infty} \ln \left[\Delta\left(i u, \mathbf{R}_{0}\right)\right] d u .
$$

The coupled modes contained in the above equation are extended over the molecules and the tip. Nevertheless, they give rise to a potential dependent on the atomic topography of the sample, although the atomic polarizability display resonances in the ultra violet. However, the virtual photons at the origin of the van der Waals interaction explains that the resulting range of potential is much shorter than the UV wavelengths because the imaginary frequencies soften the resonance effects.

From the value of the tip-sample energy, elementary partial differentiation yields the tip-sample force which we use in the next section to study the imaging SFM process by scanning rigidly the tip atoms above the adsorbed fullerene molecules. This scanning process generates a considerable amount of numerical work since the evaluation of the $3 N \times 3 N(N$ is typically above 1000 in the applications discussed below) determinant must be repeated for each position of the tip above the fullerene molecules and for a sufficient number of imaginary frequencies for the accurate 
a
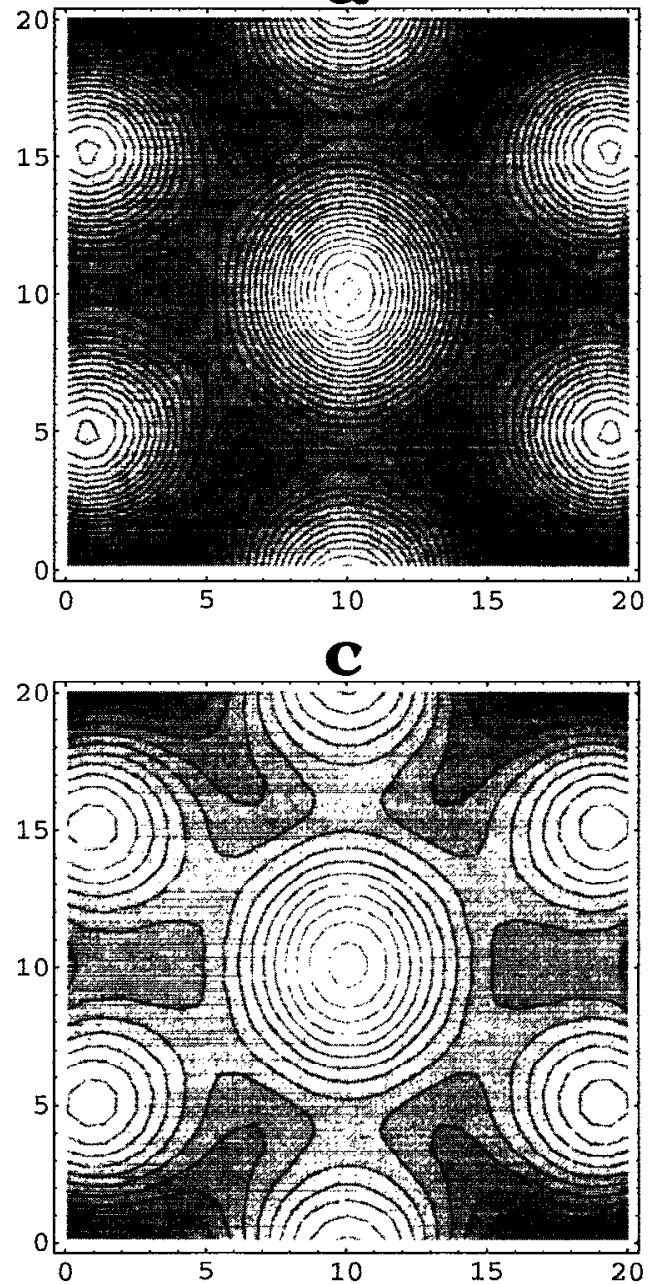

b
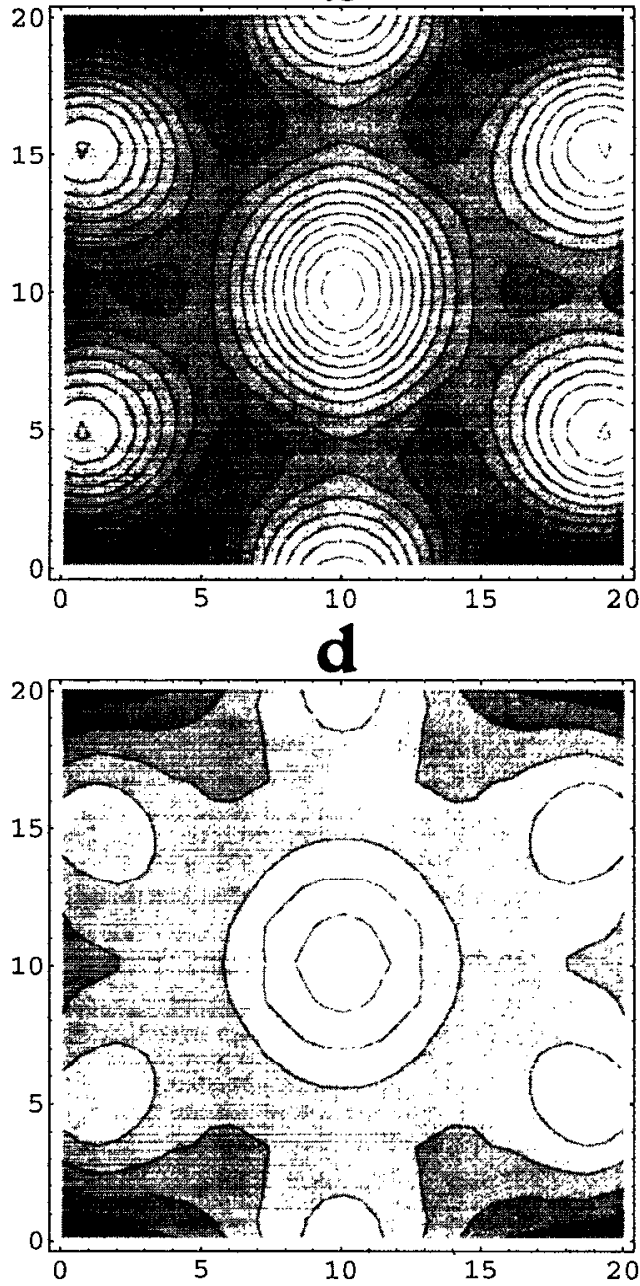

FIG. 4. Constant-distance images of a hexagonal cluster of six $\mathrm{C}_{60}$ molecules surrounding a $\mathrm{C}_{70}$. The tip scanned the substrate at the following distances: (a) $Z_{0}=14 \AA$ : the force varies from $60 \mathrm{pN}$ to $180 \mathrm{pN}$ by step of $5 \mathrm{pN}$, (b) $Z_{0}=14.5 \AA$ : the force varies from $40 \mathrm{pN}$ to $110 \mathrm{pN}$ by step of $5 \mathrm{pN}$, (c) $Z_{0}=15 \AA$ : the force varies from $26 \mathrm{pN}$ to $71 \mathrm{pN}$ by step of $3 \mathrm{pN}$, and (d) $Z_{0}=16 \AA$ : the force varies from $15 \mathrm{pN}$ to $39 \mathrm{pN}$ by step of $3 \mathrm{pN}$.

evaluation of Eq. (5). This aspect of the numerical effort decreases considerably if we evaluate first the field propagator $S_{M}\left(\mathbf{r}_{i}, \mathbf{r}_{j} ; \omega\right)$ of the surface coated with the fullerene molecules. It is obtained from the field propagator of the naked surface through a Dyson equation:

$$
\begin{aligned}
S_{M}\left(\mathbf{r}_{i}, \mathbf{r}_{j} ; \omega\right)= & S_{0}\left(\mathbf{r}_{i}, \mathbf{r}_{j} ; \omega\right)+\sum_{k=1}^{M} S_{0}\left(\mathbf{r}_{i}, \mathbf{r}_{k} ; \omega\right) \\
& \times \alpha_{k}(\omega) S_{M}\left(\mathbf{r}_{k}, \mathbf{r}_{j} ; \omega\right),
\end{aligned}
$$

where $k$ runs over the $M$ atoms which constitute the adsorbed molecules while from now $i$ and $j$ are restricted to the successive positions which will be occupied by the atoms of the tip during the scan. With the coated surface as a reference system, the evaluation of the dispersion energy involves solving Eq. (4) for the remaining $N-M$ atoms of the tip, namely

$$
\begin{aligned}
\mathbf{E}\left(\mathbf{r}_{i}\right)-\sum_{j=M+1}^{N} S_{M}\left(\mathbf{r}_{i}, \mathbf{r}_{j} ; \omega\right) \alpha_{j}(\omega) \mathbf{E}\left(\mathbf{r}_{j}\right)= & , \\
& i=M+1, \ldots N .
\end{aligned}
$$

So far, we transferred the numerical effort to Eq. (6). At first sight, solving Eq. (6) requires the inversion of a $3 M \times 3 M$ matrix so that we have apparently gained very little. In fact, the solution of Eq. (6) may also be optimized if we focus the work on the physically relevant quantities. Since we need the final values of $S_{M}\left(\mathbf{r}_{i}, \mathbf{r}_{j} ; \omega\right)$ on the grid of positions to be occupied by the atoms of the tip, we apply an iterative procedure based on the successive individual introduction of the atoms which build the molecule. The field propagator associated to the first atom deposited on the surface is extracted from

$$
\begin{aligned}
S_{1}\left(\mathbf{r}_{i}, \mathbf{r}_{j} ; \omega\right)= & S_{0}\left(\mathbf{r}_{i}, \mathbf{r}_{j} ; \omega\right) \\
& +S_{0}\left(\mathbf{r}_{i}, \mathbf{r}_{1} ; \omega\right) \alpha_{1}(\omega) S_{1}\left(\mathbf{r}_{1}, \mathbf{r}_{j} ; \omega\right) .
\end{aligned}
$$




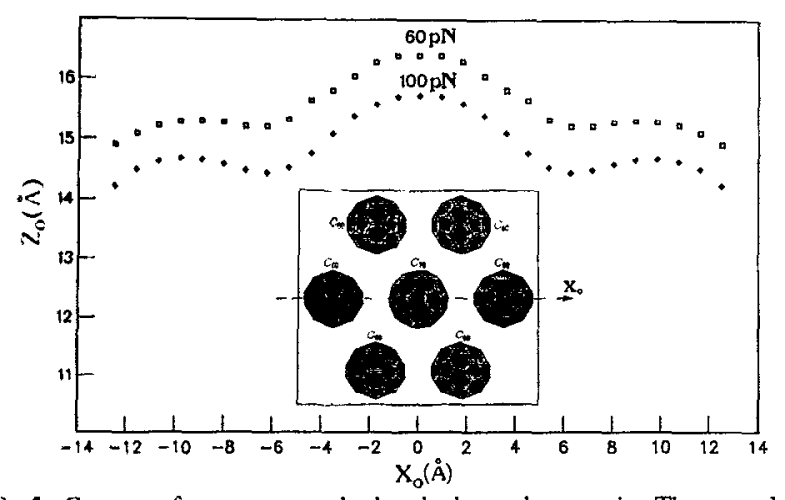

FIG. 5. Constant-force scans calculated along the $x$ axis. The sample is identical to the one described in Fig. 4 with the difference that the $C_{70}$ molecule was oriented perpendicularly to the surface. Two loading forces were considered $(60 \mathrm{pN}$ and $100 \mathrm{pN})$.

This last equation is first solved for $i=1$ through a small $3 \times 3$ system of equations and the result is then used to compute for $S_{1}\left(\mathbf{r}_{i}, \mathbf{r}_{j} ; \omega\right)$ for $i \neq 1$. The computation is limited to $j=2 \ldots . . N$ because the first atom will not be a source point at any latter stage. The same procedure is then repeated for each atom until the final construction of $S_{M}\left(\mathbf{r}_{i}, \mathbf{r}_{j} ; \omega\right)$. The computation of any intermediate propagator $S_{m}\left(\mathbf{r}_{i}, \mathbf{r}_{j} ; \omega\right)$ eliminates the $m$ th atom from the source points so that the algorithm goes forth with only $j>m$ and terminates when the $M$ th atom has acted as a source point. ${ }^{13}$

\section{SFM OF LACUNA AND $C_{70}$ AMONG AN ADSORBED $C_{60}$ ISLAND}

As a reference for later comparisons, we consider first an island of $19 \mathrm{C}_{60}$ molecules adsorbed on a flat gold surface (100) at the nodes of a hexagonal lattice (Fig. 2). The intermolecular and adsorption distances were borrowed to molecular mechanical calculations which led to 9.95 and $6.4 \AA$, respectively. ${ }^{14}$ As also observed experimentally, the twodimensional maps calculated in the attractive range do not display atomic resolution. However, each molecule is clearly distinguished. This phenomenon emphasizes the importance of collective excitations inside the fullerenes. Of course, the amplitude of the normal force increases upon approaching the sample [the force grows by $90 \mathrm{pN}$ when moving the tip from $Z=15$ to $14 \AA$, Figs. 2(b) and 2(a)]. In this range, the resulting attractive force varies sufficiently to allow the resolution of individual molecules by today's experimental devices. Indeed, approaching the tip from 15 to $14 \AA$ causes the force at the saddle points between fullerenes in the map of Fig. 2 to increase from $65 \mathrm{pN}$ up to $110 \mathrm{pN}$. The calculated force contrast, i.e., the variation of the force between the maxima and the saddle points of Fig. 2, agrees with the SFM measurements performed by Sarid et al. on the same system. ${ }^{2}$ Due to this critical dependence on the corrugation, the identification of a lacuna created by removing the central molecule is straightforward [Figs. 2(c) and 2(d)].

The stability of the image profiles were checked against the numbers of atomic layers included to model the tip. Figure 3 illustrates the evolution of the tip-sample interaction energy during a scan above an island of seven $\mathrm{C}_{60}$ molecules deposited on the gold surface. Two phenomenons were found

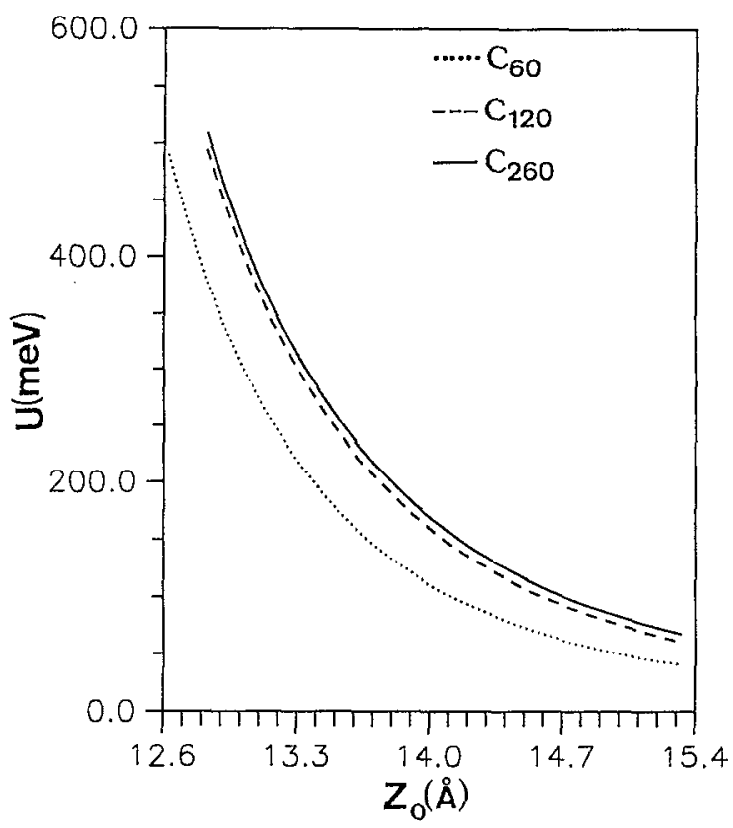

FIG. 6. Variation of the dispersion energy (meV) between a SFM (111) diamond tip and three different species of isolated fullerene molecules $\left(C_{60}\right.$. $C_{120}$, and $C_{260}$ ). The molecules are adsorbed on a (100) gold surface with an adsorption distance $D=6.4 \AA$. The calculations were performed for the tip placed right above the symmetry center of the molecule.

to occur. First, increasing the tip height contributed significantly to the signal background. As expected, due the decay of the van der Waals interaction, this effect is stabilized once the tip height is extended beyond $5 \AA$ (seven layers of $\mathrm{C}$ atoms in the tetrahedral tip). Second, even if the relative definition of the detected corrugation remained unaffected, a better absolute contrast is achieved with extending the tip. Indeed the peak to peak contrast grows from $30 \mathrm{meV}$ for a tip made of a single $\mathrm{C}$ atom [Fig. 3(a)] to about $75 \mathrm{meV}$ for tips higher than $2.5 \AA$ [four layers or $13 \mathrm{C}$ atoms in the tip, Fig. 3(b)]. Since the absolute definition of the image is obtained with a four layers tip, we can use this tip model for SFM imaging purposes. Depending on the geometrical configuration, many-body effects were responsible for at least $20 \%$ of the total interaction energy. Although this fraction is not negligible, it is less important than the proportion predicted for individual atomic systems and it does not lead to an imaging capability which differs significantly from calculations ignoring this effect (such as pairwise potential). As stated in the preceding section, the virtual nature of the coupled modes builds a long-range potential. In the attractive mode of imaging studied here, this resulting long-range potential is not sufficiently sharp to raise the atomic corrugations of the very close-packed $\mathrm{C}$ atoms. Individual $\mathrm{C}$ atoms could only be identified in the repulsive mode of imaging. Although the resonances due to many-body efrects are softened by the imaginary frequencies, they keep a complex spatial dependence (contrary to what would be obtained with a pairwise potential). In the case of the adsorbed fullerene molecules considered here, we found that the many-body effects, which are included in the present calculations, decrease the detected corrugation by modifying the amplitude of the interaction above and between the molecules. They do not 
a
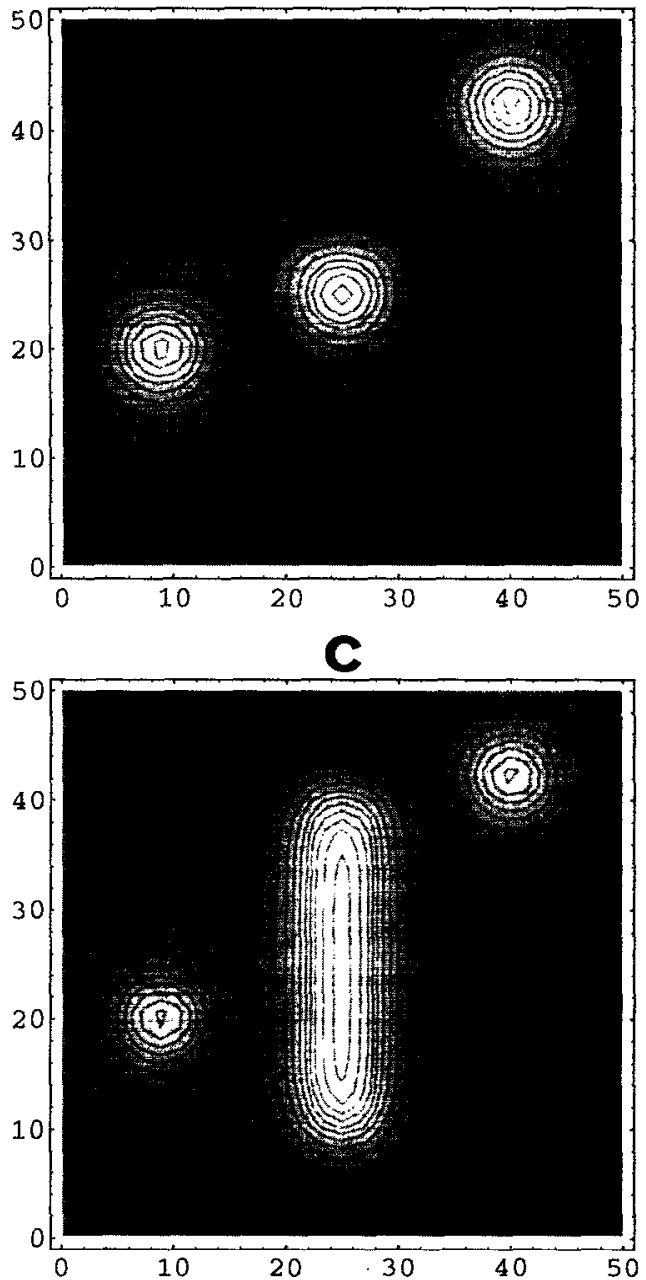

b
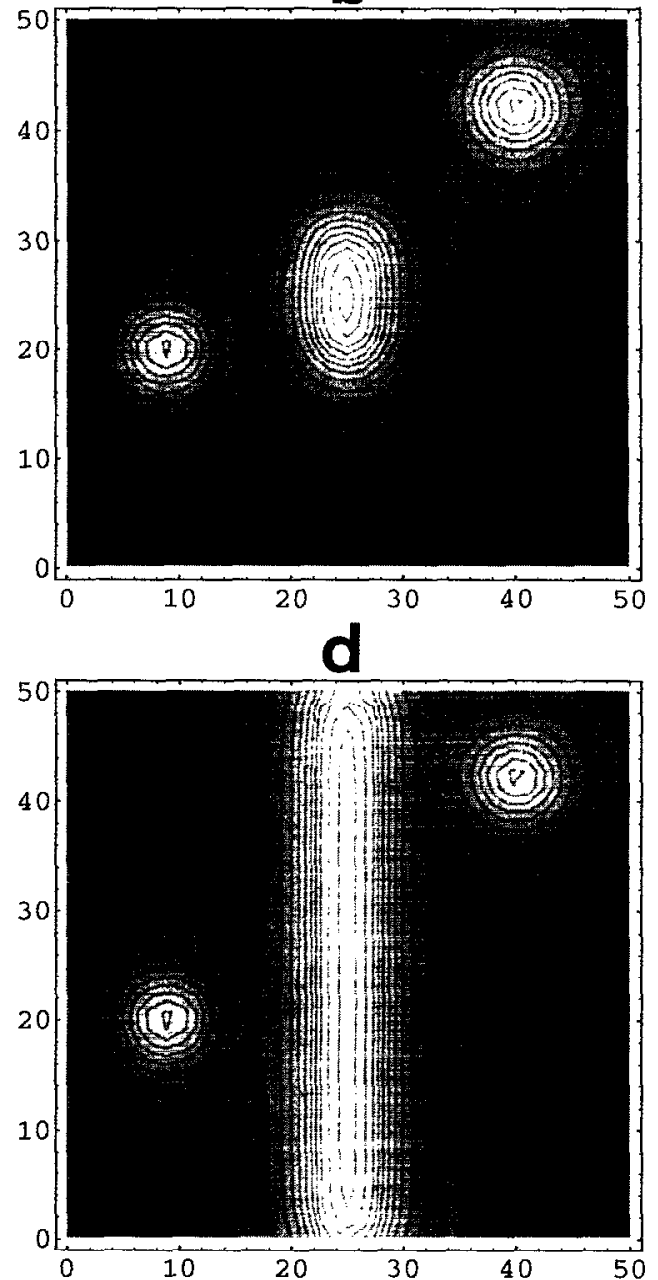

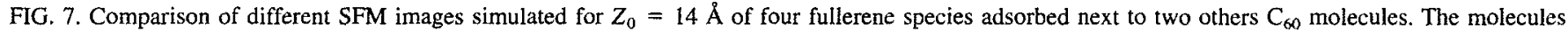

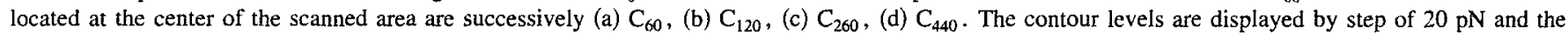
lowest value is $4.5 \mathrm{pN}$ (black areas) while the highest values (white) are respectively equal to $150 \mathrm{pN}$ (a), $211 \mathrm{pN}$ (b), $216 \mathrm{pN}$ (c), and $217 \mathrm{pN}$ (d).

however downgrade drastically the imaging capability. Including many-body effects is more important in predicting the absolute force required for the manipulation of individual fullerenes rather than in the simulation of SFM images.

We address the question of distinguishing between two fullerene species by introducing a $\mathrm{C}_{70}$ molecule at the center of the cluster. Images recorded at short distances [Figs. 4(a) and (b)] identify clearly the elongated shape of $\mathrm{C}_{70}$. Although the shape definition becomes fuzzy at larger approach distances, this last molecule could still be recognized through the magnitude of the interaction [Figs. $4(\mathrm{c})$ and $4(\mathrm{~d})$ ]. Indeed, the tip interacts more strongly with the central $\mathbf{C}_{70}$ molecule even if the axis of this $C_{70}$ molecule in the calculation lies parallel to the gold surface so that there is no height difference with the surrounding $\mathrm{C}_{60}$ molecules. The force increase is related to the larger number of atoms. This means that the tip-sample interaction is not limited to the closest atoms but integrate the contributions of all atoms inside a molecule through the collective oscillations of the charge density. As discussed in the next section, this be- havior is also found for more elongated fullerenes.

The decay of van der Waals interaction makes it possible to locate a $\mathrm{C}_{70}$ molecule which is oriented perpendicularly to the substrate by imaging in the constant force mode where the tip is scanned above the surface with a constant loading force applied to the apex. During the scanning process, this force induces an elastic deformation of the cantilever to which the tip is attached. The probe tip then follows the contour of the object while recording the images by measuring the deflection of the cantilever. The simulated trajectories of the tip apex for two loading forces are plotted in Fig. 5. The loading forces were selected to avoid the deformation of the molecules by the tip apex: in our examples, the $C$ atorn at the tip apex remained always $5 \AA$ above the upper $C$ atom of the fullerene molecules. This distance is much larger than the van der Waals radii of two $\mathrm{C}$ atoms. Our calculation demonstrates clearly that the amplitude of the $1.3 \AA$ deflection when scanning above the $C_{70}$ molecule is consistent with the difference of height of this molecule compared to $\mathrm{C}_{60}$. 


\section{IMAGING FULLERENE TUBULES}

As anticipated above when discussing the $C_{70}$ molecule embedded in the $C_{60}$ island, the size of the molecule modifies the strength of the van der Waals interaction between the tip and the molecule. This phenomenon is related to the size and the shape of the molecule. Both affect significantly the collective excitations responsible for the van der Waals interaction. We investigate this aspect further by studying the constant distance $(Z=14 \quad \AA$ ) SFM imaging of elongated fullerenes adsorbed between two $\mathrm{C}_{60}$ molecules on a gold surface (Fig. 6). These molecules are formed by the tubular assembly of $C$ atoms. We considered tubules with the same radius as $C_{(x)}$ obtained by repeating the introduction between two $C_{f(t)}$ hemispheres of the 10 atoms pattern which differentiate $C_{70}$ from $C_{60}$. In this way, elongated fullerenes closed by two $C_{60}$ caps can be achieved with various lengths. For the sake of this paper, we retained $\mathrm{C}_{120}, \mathrm{C}_{260}$, and $\mathrm{C}_{440}$ tubules in order to agree with most of the STM studies of giant fullerenes which seems to indicate that the growth of concentric tubes terminated by hemispherical caps is preferred to the formation of large concentric spherical fullerenes. ${ }^{15.16} \mathrm{We}$ computed that the force increased significantly from 150 to $211 \mathrm{pN}$ when $C_{120}$ was substituted for $C_{60}$ even if both species have the same radius when they are lying on the substrate. There is indeed more polarizable material below the tip in the case of $C_{120}$ than with $C_{60}$. Elongating further the tubule from $120 \mathrm{C}$ atoms to 260 or $440 \mathrm{C}$ atoms did not modify the maximum of the interaction energy drastically (Fig. 7). The slope of this dispersion energy with respect to the approach distance exhibits the transition of the dependence law of the van der Waals attraction force from the spherical towards the cylindrical regime. The calculations allowed to assess that this transition is already achieved for $\mathrm{C}_{120}$. Moreover, the simulated images pointed out that the interpretation of SFM scans over carbon tubules must be considered with some care. Indeed, the most important inten- sity recorded in SFM imaging is not related to different atomic elements nor to a closer tip-sample distance but definitively to the elongated shape of the fullerene tubule. The importance of collective charge density fluctuations at the origin of the van der Waals interaction explains such dependence on the shape of the molecule. ${ }^{17}$

\section{ACKNOWLEDGMENTS}

We thank A. A. Lucas for stimulating discussions. This work was performed in the framework of the Human Capital and Mobility Program initiated by the European Community. The Laboratoire de Physique Moléculaire is Unité Associée au CNRS 772.

'G. Binnig, C. F. Quate, and Ch. Gerber, Phys. Rev. Lett. 56, 930 (1986). ${ }^{2}$ D. Sarid, T. Chen, S. Howelis, M. Gallagher, L. Yi, D. L. Lichtenberger, K. W. Nebesney, C. D. Ray, D. R. Huffman, and L. D. Lamb, Ultramicrosc, 42-44, 610 (1992).

${ }^{3}$ P. Dietz, P. K. Hansma, K. Fostiropoulos, and W. Krätschmer, Appl. Phys. A 56, 207 (1993).

${ }^{4}$ P. Dietz, K. Fostiropoulos, W. Krätschmer, and P. K. Hansma, Appl, Phys. Lett. 60, 62 (1992).

${ }^{5}$ J. Viitanen. J. Vac. Sci. Technol. B 11, 115 (1993).

${ }^{6} \mathrm{C}$. Joachim, J. K. Gimzewski, in Scanning Probe Microscopies and Molecular Materials (NATO ARW, Kluwar, Dordrecht. in press).

${ }^{7}$ D. Sarid, Scanning Force Microscopy (Oxford U. P., Oxford, 1991).

${ }^{8}$ H. Tang, C. Joachim, and J. Devillers, Surf. Sci. 291, 439 (1993).

${ }^{9}$ F. London, Z. Phys. Chem. 11, 222 (1930).

${ }^{10}$ We have used a simple isotropic Lorentzian model described in H. Y. Kim, M. W. Cole, F, Toigo, and D. Nicholson, Surf. Sci. 198, 555 (1988).

"Ch. Girard and X. Bouju, J. Chem. Phys. 95, 2056 (1991).

${ }^{12} \mathrm{D}$. Langbein, Theory of van der Waals Attraction (Springer, Berlin, 1974).

${ }^{13}$ Ch. Girard, A. Dereux, and O. J. F. Martin, Surf. Sci. 295, 445 (1993).

${ }^{14} \mathrm{C}$. Chavy, C. Joachim, and A. Altibelli, Chem. Phys. Lett. 214, 569 (1993).

${ }^{15}$ M. K. Gallagher. D. Chen, B. P. Jacobsen, D. Sarid. L. D. Lamb, F. A. Tinker, J. Jiao, D. R. Huffman, S. Seraphin, and D. Zhou, Surf. Sci. Lett. 281. L335 (1993).

${ }^{10} \mathrm{M}$. Ge and K. Sattler, Science 260, 515 (1993).

${ }^{17}$ M. Schmeits and A. A. Lucas, Prog. Surf. Sci. 14, I (1983). 